\title{
Clustering as a mechanism of enhanced adsorption
}

\author{
E.V.Vakarin ${ }^{1,2}$, M.F.Holovko ${ }^{1}$ \\ 1 Institute for Condensed Matter Physics \\ of the National Academy of Sciences of Ukraine, \\ 1 Svientsitskii Str., 79011 Lviv, Ukraine \\ 2 Laboratoire de Electrochimie et Chimie Analytique, Ecole Nationale \\ Superieure de Chimie de Paris - Universite Pierre et Marie Curie, \\ 11 rue P. et M. Curie, 75231 Cedex 05, Paris, France
}

Received February 17, 2003

The role of adsorbate clustering in increasing the adsorption on crystalline surfaces is analyzed in terms of the lattice gas model combined with the results of the associative theory. The effect of the cluster size and architecture in equilibrium and transport properties is studied. Some conjectures related to $\mathrm{HCl}$-ice interfaces are drawn.

Key words: adsorption, clustering, chemical diffusion

PACS: $68.45 .-v$

\section{Introduction}

Adsorption from gaseous or condensed phases onto solid surfaces is a phenomenon, which has numerous technologically important applications $[1,2]$. The process is driven by several factors which are usually competing. These include the adsorbatesubstrate, the substrate-substrate and adsorbate-adsorbate interactions, which were shown $[3,4]$ to result in a remarkable interface restructuring, especially when the substrate is not absolutely rigid. It is important that at fluid-solid interfaces the fluid (adsorbate) acts as a thermodynamic subsystem [5] whose properties are determined from the requirement of the solid-fluid equilibrium. This means that the adsorbate has an additional (energetic and entropic) impact from its bulk, which induces a correlation for the adsorbed species. The correlation through the bulk is particularly pronounced when the pair interaction in the fluid is attractive enough to induce the clustering [6,7]. Then, the intra-cluster correlation, arising due to the cluster connectivity, enhances the adsorption in comparison with the monomeric case. The effect takes place at a rather low adsorbate density in the interfacial region, that is, when the cluster-cluster repulsion is negligible. This mechanism has 
been shown [8] to be responsible for a remarkable increase of the surface coverage at low polymer concentrations.

In this paper we study the clustering as a mechanism of increasing the adsorption, focusing on how this effect depends on the adsorbate density, cluster size and cluster shape (linear chains or network-like aggregates). We first discuss the equilibrium characteristics and then analyze the kinetic properties.

\section{Adsorption of clusters}

\subsection{General formulation}

We consider a model of a clustering fluid-solid interface. The fluid consists of associating hard spheres with the diameter $\sigma$ and bulk number density $\rho$, so that the packing fraction is $\eta=\pi \rho \sigma^{3} / 6$. The association is mediated by the site-site interaction responsible for a formation of clusters. Depending on the specificity of the site-site attraction, different shape clusters can be formed (e.g. dimers, linear polymer chains, network-like aggregates, star polymers). In this study we restrict ourselves to the cases of linear chains and four-fold coordinated network-like clusters. Details of the related potential models can be found in [6-8]. Therefore, the fluid is characterized by its packing $\eta$ and mean cluster size $S$. The latter is the number of hard sphere monomers belonging to a cluster.

The fluid is near a hard wall (located at $z=0$ ) on which there is a lattice of $N$ sticky sites (lattice spacing is $d$ ), modelling a crystalline structure. The adsorption potential $U_{s}\left(\mathbf{r}_{i}\right)$ is defined by

$$
\exp \left(-\beta U_{s}\left(\mathbf{r}_{i}\right)\right)=1+\lambda \sum_{\mathbf{R}_{m}} \delta\left(\mathbf{R}_{i}-\mathbf{R}_{m}\right) \delta(z)
$$

where $\mathbf{R}_{i}$ is a projection of $\mathbf{r}_{i}$ onto the wall, $\mathbf{R}_{m}$ is a position of a lattice site and $\lambda$ is the stickiness parameter. This singular one-body potential allows one to perform an exact integration in the partition function

$$
Z=Z_{\text {ref }} \sum_{n=0} \frac{\lambda^{n}}{n !} \sum_{\mathbf{R}_{n}} \rho_{n}^{\mathrm{ref}}\left(\mathbf{R}_{1}, \ldots, \mathbf{R}_{n}\right),
$$

where $Z_{\text {ref }}$ is the partition function for the same system but without the adsorbing potential, and $\rho_{n}^{\text {ref }}\left(\mathbf{R}_{1}, \ldots, \mathbf{R}_{n}\right)$ is the $n$-body distribution function taken at the positions of the lattice sites. In such a way we have an infinite series on $\lambda$ including the correlations of all orders for the reference state. If only pair interactions are important, then the problem can be mapped onto the lattice gas model $[9,10]$

$$
\Xi=Z / Z_{\mathrm{ref}}=\sum_{t_{i}} \exp \left(-\beta H_{\mathrm{LG}}\right)
$$

with the Hamiltonian

$$
H_{\mathrm{LG}}=\sum_{i j} W\left(\mathbf{R}_{i}, \mathbf{R}_{j}\right) t_{i} t_{j}-\sum_{i} \mu\left(\mathbf{R}_{i}\right) t_{i}
$$


in which $t_{i}$ is a set of occupation numbers. The chemical potential $\mu\left(\mathbf{R}_{i}\right)$ and pair interaction $W\left(\mathbf{R}_{i}, \mathbf{R}_{j}\right)$ are closely connected with the properties of the fluid in the bulk phase. Namely,

$$
\begin{aligned}
\beta \mu\left(\mathbf{R}_{i}\right) & =\ln \left(\lambda \rho_{1}^{\mathrm{ref}}\left(\mathbf{R}_{i}, \sigma / 2\right)\right), \\
\beta W\left(\mathbf{R}_{i}, \mathbf{R}_{j}\right) & =-\ln \left(g_{2}^{\mathrm{ref}}\left(\mathbf{R}_{i}, \mathbf{R}_{j}\right)\right),
\end{aligned}
$$

where $\rho_{1}^{\text {ref }}\left(\mathbf{R}_{i}\right)$ and $g_{2}^{\text {ref }}\left(\mathbf{R}_{i}, \mathbf{R}_{j}\right)$ are respectively the one-body and pair correlation functions for the reference state (i.e., a state without specific adsorption) - the hard wall problem. The latter is independent of the adsorption site position, and if $\sigma \approx d$ then we deal with the contact values $\rho_{1}(\sigma / 2)$ and $g_{2}(\sigma)$. In the Percus-Yevick approximation they are given by $[6,8]$

$$
\begin{aligned}
\rho_{1}(\sigma / 2) & =\rho\left[\frac{1+2 \eta}{(1-\eta)^{2}}-\frac{S-1}{S} \frac{1}{1-\eta}\right] \\
g_{2}(\sigma) & =\frac{1+\eta / 2}{(1-\eta)^{2}}-\frac{S-1}{S} \frac{1}{1-\eta}+\frac{C}{\eta}\left[\frac{S-1}{S}\right]^{2} .
\end{aligned}
$$

For both functions, the first terms correspond to the hard sphere packing effects which dominate with the increase of $\eta$. The second terms describe the depletion with the increase of $S$ due to the cluster-wall or cluster-cluster repulsion. The last term in $g_{2}(\sigma)$ is responsible for the intramolecular correlation inside a cluster. At fixed $\eta$ and $S$ the intensity of this correlation is given by the coefficient $C$. For chains $C=1 / 24$ and for networks $C=1 / 16$. Therefore, in the approximation considered here, the network-like clusters differ from linear chains only by the strength of the intramolecular correlation. The latter dominates at low densities when the clustercluster contacts are rather rare and the monomers correlate mainly through the cluster connectivity. Note, however, that the mean cluster size $S$ is chosen as an external variable. This means that we do not consider the clustering process itself, assuming that the cluster composition remains fixed in the course of adsorption. This assumption is valid if the adsorption is not dissociative and the adsorption rate dominates the rate of dissociation in the fluid bulk. In general, $S$ should be considered as a function of density and temperature. Then, equations (7) and (8) remain applicable with $S=S(\eta, T)$. In this case the difference between the chains and network clusters would be remarkable. In particular, for networks, $S$ could diverge signalling the percolation threshold $[6,7]$.

\subsection{Mean-field solution}

Having determined the bulk input to the lattice gas Hamiltonian we can find the mean-field approximation for the free energy, which, after minimization, gives the relation for the chemical potential at the equilibrium

$$
\beta \mu=q \beta W \theta+\ln \left(\frac{\theta}{1-\theta}\right),
$$




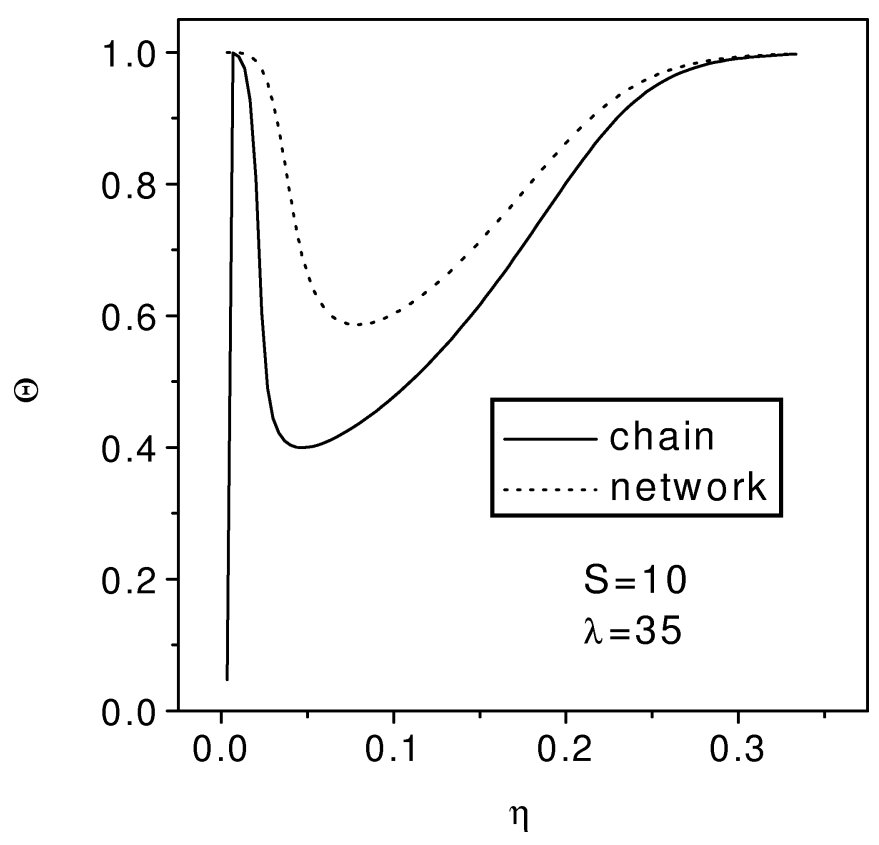

Figure 1. Surface coverage $\theta$ as a function of the bulk packing fraction $\eta$ for chains and network-like clusters of the same mean size $S$.

where $\theta=\left\langle\sum_{i} t_{i}\right\rangle / N$ is the coverage and $q$ is the lattice coordination number. Equation (9) has been solved numerically in order to determine the coverage $\theta$ as a function of density $\eta$ and cluster size $S$. Depending on $\eta$ and $S, g_{2}(\sigma) \geqslant 1$ or $<1$ and consequently the lateral interaction $\beta W=-\ln g_{2}(\sigma)$ is either attractive or repulsive. For negative $\beta W$ the system exhibits two-dimensional criticality of the gas-liquid type under the conditions $\left.q \beta W\right|_{\mathrm{c}}=-4$ and $\left.2 \mu\right|_{\mathrm{c}}=\left.q W\right|_{\mathrm{c}}$. Then, for given surface activity $\lambda$ and $S$ the coverage becomes discontinuous at some critical density $\eta_{\mathrm{c}}$. Note that $g_{2}(\sigma)$ is a nonmonotonic function of $\eta$ as a result of the competition between the inter- and intramolecular correlations. Therefore, the critical condition can be reached in two ways. First is the case of low surface activity $\lambda$ and high density $\eta$. Then we deal with a cluster condensation induced by the packing effects. This effect has been extensively analyzed $[9,10]$ and we focus on the second way, namely - the case of high surface activity and low density. Then the cluster condensation is induced by the intramolecular correlation. In other words, at low densities when the cluster-cluster contacts are negligible, the adsorption of one bead promotes the adsorption of the entire cluster. This cooperative effect has been confirmed experimentally [8]. The role of clustering is remarkable as the coverage increases by several orders of magnitude in comparison with the case of monomeric adsorption $(S=1)$.

In figure 1 the coverage is plotted as a function of density. As is discussed above, $\theta$ is a nonmonotonic function of $\eta$. Three distinct domains are clearly seen. At low $\eta$ the coverage grows sharply exhibiting the cluster condensation transition due to the intramolecular correlation. Then, at intermediate densities the cluster-cluster repulsion leads to a decrease of $\theta$. At high densities the coverage increases again due 


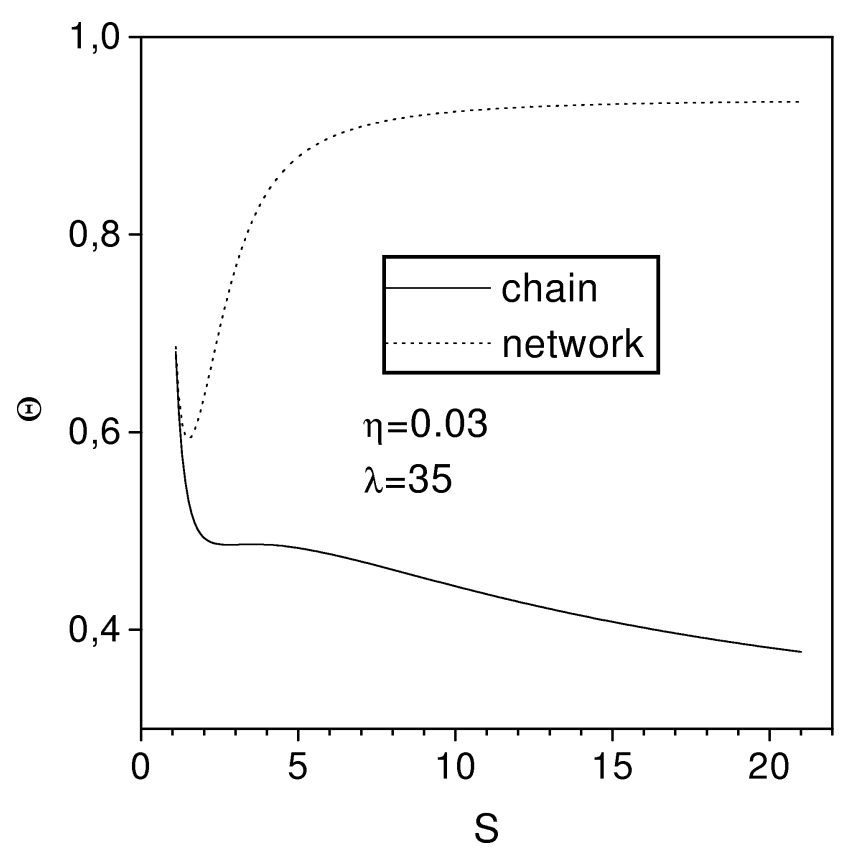

Figure 2. Surface coverage $\theta$ as a function of the mean cluster size $S$ for chains and network-like clusters.

to the packing effects. It is seen that the network-like clusters are more efficient in increasing $\theta$ than the chains of the same size.

In figure $2 \theta$ is plotted as a function of $S$ at $\eta=0.03$, which belongs to the region where the cluster-cluster repulsion decreases the coverage. For network-like clusters $\theta$ grows with $S$ due to the increasing role of the intramolecular correlations. On the contrary, the chain coverage decreases in a broad range of cluster sizes since the intramolecular correlation is weaker than that in the network. This once again emphasizes the efficiency of the network in increasing the coverage.

\section{Kinetic properties}

Based on the equilibrium properties we can investigate the kinetic characteristics with the purpose to realize the role of the clustering effects. Starting from equation (9) the chemical diffusion coefficient can be found as [11]

$$
D / D_{0}=\theta(1-\theta) \frac{\mathrm{d}(\beta \mu)}{\mathrm{d} \theta}=1+q \beta W \theta(1-\theta),
$$

where $D_{0}$ is the single site hopping. It is seen that for attractive interaction $(\beta W<0)$ the diffusion coefficient decreases. Taking $\theta$ as a solution of equation (9) we analyze the behavior of $D / D_{0}$ as a function of $\eta$ at different $S$ for the case of polymer chains. This is depicted in figure 3. As expected, at low densities the diffusion is suppressed due to the cluster condensation effect. At intermediate densities the diffusion coefficient $D / D_{0}$ is even higher than that of monomers $(S=1)$ due to the increasing role of the cluster-cluster repulsion. This may seem rather strange. 


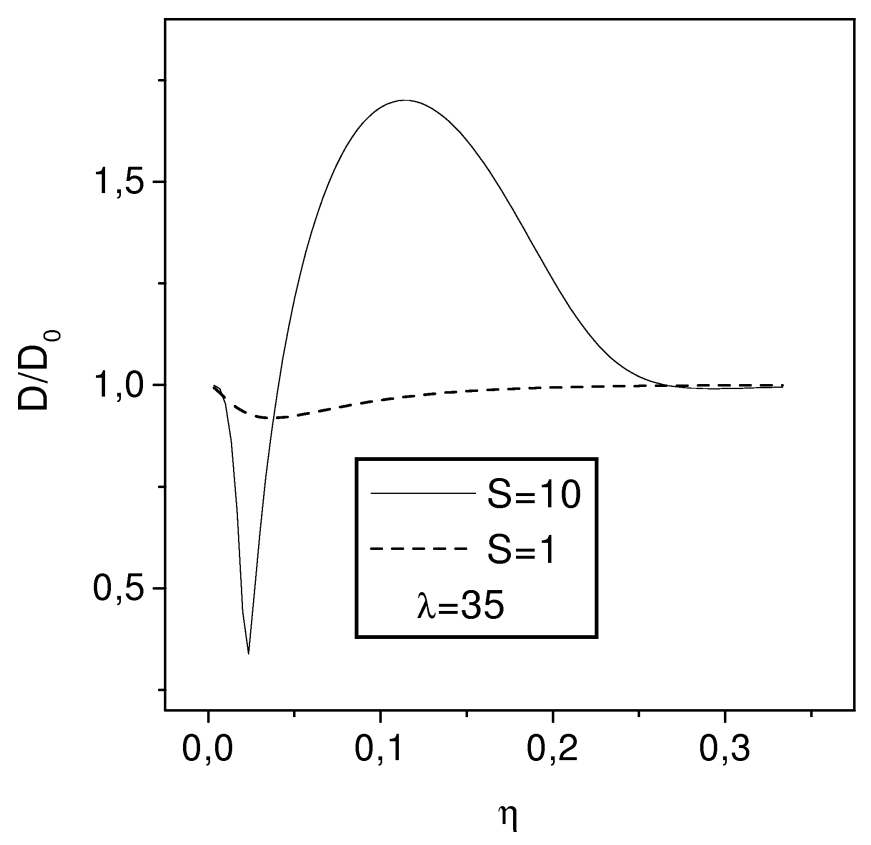

Figure 3. The surface chemical diffusion coefficient for monomers and chains as a function of the bulk packing fraction.

In this respect we have to point out that we deal with a "single-site" transport in the presence of the clustering, but not with the motion of clusters. Moreover, the monomer coverage is much higher in this domain of densities. Hence, the monomer transport is essentially blocked by the factor $1-\theta$, while there is enough free space for the clusters to diffuse. Note also that we operate with the single site hopping $D_{0}$, but the cluster hopping should decrease with $S$ (e.g. like $D_{0} / S$ ) such that the absolute value of $D$ (at the same coverage) for clusters would be reduced in comparison with that for monomers. Nevertheless, it is clear that the functional dependence of $D$ vs $\eta$ in the presence of clustering is different from that of monomers.

As we have seen above the clustering significantly enhances the adsorption, especially at low densities. The clustering itself is a balance of two competing processes evolving in time, i.e. formation of bonds between the monomers and their destruction (spontaneously or under external fields). Obviously, the stability of such a cluster adlayer depends upon the average number of bonds $n(t)$ per monomer. In the meanfield spirit such a balance is given by the following kinetic equation

$$
\tau \frac{\partial n(t)}{\partial t}=a\left[n_{m}-n(t)\right]-b n(t)
$$

where $\tau$ is the characteristic time scale, the coefficients $a$, and $b$ are the intensities of the bond formation and destruction, respectively, $n_{m}$ is the maximal number of bonds per monomer (e.g., $n_{m}=4$ for network-like clusters and $n_{m}=2$ for polymer chains). The solution with the initial condition $n(t=0)=n_{0}$ is given by

$$
n(t)=n_{m} \frac{a}{a+b}\left[1-\exp \left(-\frac{(a+b) t}{\tau}\right)\right]+n_{0} \exp \left(-\frac{(a+b) t}{\tau}\right) .
$$


It is clear that the asymptotic behavior does not depend on the initial condition

$$
n_{a}=n(t \rightarrow \infty)=n_{m} \frac{a}{a+b}
$$

Nevertheless, depending upon whether $n_{0}<n_{a}$ or $n_{0}>n_{a}, n(t)$ approaches $n_{a}$ either from below (partial self-healing) or from above (partial destruction). Therefore, the clustering-mediated enhancement of the adsorption could decrease with the time increase depending upon which of the two cases is realized. This might be essential for analyzing the adsorption kinetics.

\section{Conclusion and implications}

It is shown that the clustering in the interfacial region enhances the adsorption by several orders of magnitude due to an increasing role of the intracluster correlation. This effect occurs at low adsorbate densities in the interfacial region, that is, when the cluster-cluster repulsion is negligible. It is demonstrated that the network-like clusters are more efficient in increasing the coverage than the linear chains of the same size. The functional dependence of the chemical diffusion coefficient on the adsorbate interfacial density in the presence clustering is found to be different from that for monomers.

Similar enhancement effect could take place in the course of $\mathrm{HCl}$ adsorption on ice surfaces [12]. It is known that $\mathrm{HCl}$ forms a hydrogen bond with ice. Therefore, one can expect an attraction (with the hydrogen bond strength $\approx 20 \mathrm{~kJ} /$ mole) between $\mathrm{HCl}$ and water during the $\mathrm{HCl}$-vapor co-adsorption [13]. As we have shown [14], such an assumption is compatible with experimental data on the $\mathrm{HCl}$ coverage and adsorption energy. At stratospheric conditions (low temperatures and pressures), resembling the low density region in our model, the attraction could result in $\mathrm{HCl}$ water clustering over the surface of ice. Then the adsorption would increase through the two-dimensional condensation transition. This conjecture is partially coherent with the encapsulation scenario [13] studied in a dynamic context. Therefore, a detailed study of the $\mathrm{HCl}$-water interaction would be much helpful in analyzing the clustering. The latter enhances the adsorption and also is essential for the $\mathrm{HCl}$ ionization, providing a link between these two processes.

\section{Acknowledgements}

This work was supported in part by INTAS (Grant INTAS99-01162).

\section{References}

1. Fleer G.J., Lyklema J. - In: Adsorption from Solution at Solid/Liquid Interface. G.D.Parfitt and C.H.Rochester Eds., New York, Academic press, 1983.

2. Kawaguchi M., Takahashi A. // Adv. Colloidal and Interface Science, 1992, vol. 37, p. 219. 
3. Vakarin E.V., Filippov A.E., Badiali J.P. // Phys. Rev. Lett., 1998, vol. 81, p. 3904.

4. Vakarin E.V., Filippov A.E., Badiali J.P. // Surf. Sci., 1999, vol. 422, p. L200.

5. Vakarin E.V., Badiali J.P. // Phys. Rev. B, 1999, vol. 60, p. 2064.

6. Vakarin E.V., Duda Yu., Holovko M.F. // J. Chem. Phys., 1997, vol. 107, p. 5569.

7. Vakarin E.V., Duda Yu., Holovko M.F. // J. Stat. Phys., 1997, vol. 88, p. 1333.

8. Voronov A., Luzinov I., Minko S., Sidorenko A., Vakarin E., Holovko M. // Macromolecules, 1997, vol. 30, p. 6929.

9. Badiali J.P., Blum L., Rosinberg M.L. // Chem. Phys. Lett., 1986, vol. 129, p. 149.

10. Holovko M. F., Vakarin E. V. // Chem. Phys. Lett., 1994, vol. 230, p. 507.

11. Vakarin E.V., Holovko M.F. // Chem. Phys. Lett., 2001, vol. 349, p. 13.

12. Solomon S., Garcia R.R., Rowland F.S., Wuebbles D.J. // Nature, 1986, vol. 321, p. 755 .

13. Gertner B.J., Hynes J.T. // Science, 1996, vol. 271, p. 1563.

14. Kapko V.I., Vakarin E.V., Holovko M.F. Preprint of the Institute for Condensed Matter Physics, ICMP-02-26E, Lviv, 2002, 7 p.

\title{
Кластеризація як механізм посилення адсорбції
}

\author{
Е.В.Вакарін ${ }^{1,2}$, М.Ф.Головко ${ }^{1}$ \\ 1 Інститут фізики конденсованих систем НАН України, \\ 79011 Львів, вул. Свєнціцького, 1 \\ 2 Лабораторія електрохімії та аналітичної хімії, Вища національна \\ школа хімії в Парижі - Університет П'єра і Марії Кюрі, \\ Франція, Париж, вул. П'єра і Марії Кюрі, 11
}

Отримано 17 лютого 2003 р.

В рамках моделі граткового газу, доповненої результатами асоціативної теорії, аналізується роль кластеризації адсорбату на зростання адсорбції на кристалічній поверхні. Вивчається вплив розмірів і архітектури кластера на рівноважні та транспортні властивості. Зроблені певні передбачення відносно міжфазної області $\mathrm{HCl}$-лід.

Ключові слова: адсорбція, кластеризація, хімічна дифузія

PACS: $68.45 .-v$ 